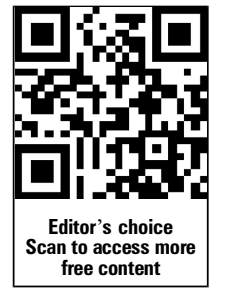

For numbered affiliations see end of article.

\section{Correspondence to}

Dr Jennifer C Davis,

Department of Health Care and Epidemiology, University of British Columbia, 828 West 10th Ave, Vancouver, British Columbia, Canada V5Z 1M9; jennifer.davis@ubc.ca

Accepted 1 April 2014

\title{
2014 Consensus Statement from the first Economics of Physical Inactivity Consensus (EPIC) Conference (Vancouver)
}

\author{
Jennifer C Davis, ${ }^{1,2}$ Evert Verhagen ${ }^{3}$ Stirling Bryan, ${ }^{2}$ Teresa Liu-Ambrose, ${ }^{4}$ \\ Jeff Borland, ${ }^{5}$ David Buchner, ${ }^{6}$ Marike RC Hendriks, ${ }^{3,7}$ Richard Weiler, ${ }^{8}$ \\ James R Morrow Jr, ${ }^{9}$ Willem van Mechelen, ${ }^{3}$ Steven N Blair, ${ }^{10}$ Mike Pratt, ${ }^{11}$ \\ Johann Windt, ${ }^{12}$ Hashel al-Tunaiji, ${ }^{4}$ Erin Macri, ${ }^{4}$ Karim M Khan, ${ }^{13,14}$ The EPIC Group
}

\section{ABSTRACT}

This article describes major topics discussed from the 'Economics of Physical Inactivity Consensus Workshop' (EPIC), held in Vancouver, Canada, in April 2011. Specifically, we (1) detail existing evidence on effective physical inactivity prevention strategies; (2) introduce economic evaluation and its role in health policy decisions; (3) discuss key challenges in establishing and building health economic evaluation evidence (including accurate and reliable costs and clinical outcome measurement) and (4) provide insight into interpretation of economic evaluations in this critically important field. We found that most methodological challenges are related to (1) accurately and objectively valuing outcomes; (2) determining meaningful clinically important differences in objective measures of physical inactivity; (3) estimating investment and disinvestment costs and (4) addressing barriers to implementation. We propose that guidelines specific for economic evaluations of physical inactivity intervention studies are developed to ensure that related costs and effects are robustly, consistently and accurately measured. This will also facilitate comparisons among future economic evidence.

\section{INTRODUCTION}

Healthcare resources are finite and demands on public health expenditures are rising. ${ }^{1}{ }^{2}$ Hence, policy and decision-makers are forced to make choices for resource allocation based on competing alternatives. Such alternatives are suggested by the Global Advocacy for Physical Activity Council that identified seven 'best investments' for promoting physical activity based on existing global evidence. ${ }^{3}$ These include (1) school programmes; (2) transportation policies and systems; (3) urban design and infrastructure; (4) physical activity built into primary prevention strategies; (5) public education; (6) community programmes and (7) 'sport for all'. ${ }^{3}$ Information about the costs and effects of policy alternatives are important. This article discusses these issues as they apply to the health and economic burden of physical inactivity. Specifically, we (1) document that physical inactivity is a major public health problem and that effective interventions exist to address these problems; (2) document the potential of cost mitigation from effective interventions, given high healthcare and loss of productivity costs due to inactivity; (3) identify key challenges in building economic evidence and (4) call for more emphasis to be paid to economic evaluation of solutions to the inactivity pandemic.

\section{Section 1: The prevalence of physical inactivity - it is a pandemic}

Physical inactivity is one of the most pressing healthcare issues of the 21 st century. It is the fourth leading cause of global mortality and is among the top 10 leading causes of death and disability in moderate-to-high income countries. ${ }^{1}$ Briefly, physical activity is defined as a behaviour involving human movement that results in physiological attributes which may include increased energy expenditure and improved physical fitness. ${ }^{4}$ Physical inactivity is the absence of such behaviour. Decreasing the prevalence of physical inactivity by $25 \%$ would avert 1.3 million deaths annually. ${ }^{5}$ In 2003 , the overall prevalence of physical inactivity worldwide was $31.1 \%$ (95\% CI 30.9 to 31.2$){ }^{6}$ Physical inactivity is also greater in high-income countries. ${ }^{6}$ The population attributable fraction of physical inactivity of major non-communicable diseases (ie, coronary heart disease; breast and colon cancers and type 2 diabetes) is substantial and emphasises the importance of physical inactivity as a major public health risk factor. ${ }^{5}{ }^{8-10}$ Specifically, the population attributable fraction of physical inactivity was $6 \%$ for coronary heart disease, $10 \%$ for breast cancer, $10 \%$ for colon cancer and $7 \%$ for type 2 diabetes in the USA. ${ }^{5}$

\section{Section 2: The economic burden of physical inactivity}

A large proportion of healthcare costs are predominantly driven by diseases caused by unhealthy lifestyle including physical inactivity. There are consistent data that $1-4 \%$ of total direct healthcare costs are attributable to physical inactivity. ${ }^{11}$ In the UK (2009), the total estimated costs for physical inactivity was $£ 8.3$ billion (population $\sim 50$ million). ${ }^{12}$ Of note, this estimate was based on only four chronic diseases linked to physical inactivity; therefore, the actual costs when including all diseases linked to inactivity will be much higher. ${ }^{12}$

However, considering only the direct costs of inactivity substantially under-represents its true economic burden. More specifically, studies have also demonstrated the indirect costs attributable to physical inactivity were more than double the associated direct costs. $^{11}$ Productivity costs also 
represent a larger proportion in comparison to direct costs. Hence, the costs of physical inactivity are large; so there is potential for major cost savings.

Until now, productivity costs are often not included in the cost of physical inactivity estimates leading to a substantial under-representation of the true burden of physical inactivity, especially in worksite programmes. ${ }^{2}$ The issues of whether to include both direct and productivity costs warrants scrutiny. ${ }^{13}$ Productivity is a measure of the rate at which a work task is performed. In ascertaining the cost of physical inactivity, specific examples of productivity costs may include loss of time at work (absenteeism), reduced productivity while at work (presenteeism) and costs of care. Lost productivity can be attributable to absenteeism or presenteeism where employees are either absent from work or they are functioning at a lower rate while at work due to health-related conditions. Specific examples of questionnaires that ascertain an individual's productivity include the (1) Health and Work Questionnaire, (2) Work Ability Index and (3) Work Limitations Questionnaire. Specifically, these questionnaires are suitable for exploring the relationship between physical activity and presenteeism. ${ }^{14}$ Reasons for including productivity costs are that (1) legitimate productivity costs contribute to the goal of a 'fully costed' model and, (2) in certain settings (ie, the workplace) productivity costs are the major contributor to the cost of illness estimates. Arguments against including productivity costs are that (1) there is no consensus about how to estimate and value productivity loss and the methodological quality of available instruments remains to be studied $^{15}$; (2) there is a risk on double counting as the value of improved health may already include the value of increased productivity; (3) it depends on the objective and perspective of the study and (4) the issue of equity needs to be considered where those who 'work' essentially have their disease valued more highly than those who do not. Of note, for the purpose of valuing the costs in relation to physical inactivity, this argument may not hold when we are estimating the overall public health burden of physical inactivity because we do not value a disease at an individual level. At a societal level, it is important to recognise that if an individual is more productive and thus earns more, some of this revenue will go to the government as tax revenue and will be subsequently redistributed by the government benefiting the rest of society in some way. Hence, the societal value of identifying cost-effective intervention strategies, which combat physical inactivity is critical, especially given competing demands on healthcare resources. ${ }^{16} 17$

\section{Section 3: Behavioural intervention strategies can prevent and mitigate physical inactivity}

Given the substantial economic burden of physical inactivity, it should be asked, 'Can this cost be mitigated?' The solution, physical activity, can be delivered in numerous different forms (frequency, intensity, type and duration), for different people and given by different individuals (including but not limited to brief physician intervention or as part of a physical activity programme in schools or for older people). ${ }^{2}$ Thus, we briefly review the existing evidence for the different forms of this therapy. We aim to mirror the approach of a pharmacoeconomic review of an oncology drug, which would include the level of effectiveness of that drug.

Physical activity (including aerobic and musculoskeletal fitness) is a potentially inexpensive treatment for physical inactivity that has demonstrated benefits on 39 diseases or health conditions. ${ }^{18}$ A systematic review detailed the results of 29 randomised controlled trials (RCTs) aimed at promoting physical activity or combatting inactivity. ${ }^{19}$ The behavioural interventions used were heterogeneous in nature and included individual or group counselling, self-directed or prescribed physical activity; supervised or unsupervised physical activity; home-based or facility-based physical activity; social interaction or support in person; telephone interaction or support; written material; and self-monitoring. The interventions were delivered by individuals from a variety of health backgrounds including physicians, nurses, health educators, physical therapists, counsellors, exercise leaders and peers. The authors concluded that behavioural interventions aimed at promoting physical activity have a positive (OR greater than 1) and moderate effect (OR between 2 and 11) on self-reported physical activity. More specific conclusions were not drawn due to heterogeneity in interventions, outcome measures and analytic techniques. Given that the effects were positive, the impact such interventions would have on public health may be substantial. Specifically, one study found that large gains among individuals who were sedentary were equally as valuable as the small gains experienced by individuals who were already active. ${ }^{20}$ This provides support for all positive gain experienced at a population level.

\section{Section 4: Economic evaluations of physical inactivity interventions: a scarce, but essential tool to inform policy decisions}

In our current landscape of finite and limited resources, policy makers must make informed decisions about how to best use the available resources. Economic evaluations-cost-benefit, cost-effectiveness (CEA) and cost-utility analyses-are essential elements needed to guide expenditure decisions. To the best of our knowledge, there is a limited evidence base of health economic enquiry from RCTs of interventions aimed at ameliorating physical inactivity. Most recently, an economic analysis was conducted from a systematic review of effective physical activity interventions. $^{21}$ Here, we summarise several main findings. Considering interventions that were inexpensive to deliver, the most cost-effective strategies were for point-of-decision prompts; however, these strategies had small effect sizes adding only $0.2 \%$ of minimum recommended physical activity levels. Other interventions that were noted to have more potential to be cost effective at a population level were environmental interventions and interventions targeted at general practitioners. ${ }^{22}$ In summary, such inexpensive interventions lead to minimal effects and minimal costs which are key factors for population level interventions. In contrast, the expensive interventions were the least cost effective. One systematic review demonstrated that effective behavioural interventions were able to promote physical activity costing approximately $€ 800$ annually. ${ }^{22}$ Another systematic review detailed interventions consisting of high-intensity 'individually adapted behaviour change' and 'social support' programmes. ${ }^{21}$ These interventions had the largest effect sizes, adding $35-43 \%$ of recommended physical activity from their baseline values. Thus, expensive interventions generally lead to larger effects at a greater cost. ${ }^{23} 24$

Despite these promising initial results, much more work needs to be carried out using RCT methodology coupled with rigorous economic evaluation methodology. This will enable us to understand the differential effects of intervention type, duration and frequency in order to determine which strategy provides the best value for money-evaluating cost and consequences. Another important factor to consider using RCT methodology is the choice of outcome. ${ }^{25}$ It may not be appropriate to judge a physical activity trial using the standard medical evidence criteria that invariably are concerned with 
dichotomous outcomes (success/failure). ${ }^{25}$ Increased physical activity has multiple benefits that are best captured using multiple in a continuous fashion (ie, very beneficial, beneficial, moderately beneficial, not beneficial and moderately harmful). ${ }^{25}$ Given the inherent methodological challenges present in interpreting and synthesising RCT evidence, it is not surprising that this further complicates interpretation of health economic evidence. Critically relevant to the current evidence base is further identification and discussion of methodological challenges related to conducting economic evaluations alongside clinical trials (RCT) of behavioural interventions aimed at combatting physical inactivity.

By way of interim summary, if policy makers were to ask the exercise medicine community-what is the evidence to indicate that interventions can provide value for money? The answer would be that to date we have limited high-quality health economic evidence for accurately ascertaining the value for money of various intervention strategies targeted at physical inactivity. Briefly, interventions that are cost effective at a population level will be different than individually tailored interventions intended for specific subpopulations. Important articles have demonstrated that at a population level, we can provide the best value for money using strategies that have low implementation costs and demonstrate modest gains in physical activity or general health outcomes. ${ }^{26-28}$ It may be that expensive interventions are best targeted at selected groups where they will demonstrate the most effectiveness. ${ }^{23} 2429$ Of note, different methods to determine which interventions can provide the best value for money may likely produce different results. As such, it is important that policy makers ensure their policy questions align with the appropriate research methods. For example, Cobiac et $a l^{28}$ model best value for money using a lifetime time horizon. Whereas, other studies may use a systematic review approach of CEA that were coupled with clinical trials using only 1 -year or 2 -year time horizons. ${ }^{21}$ Hence, we first need to identify and address the existing methodological challenges specific to conducting economic evaluations of physical inactivity interventions.

\section{Section 5: Methodological challenges for health economists in sport and exercise medicine \\ Challenges in conducting a concurrent economic evaluation}

Trials with physical inactivity as an outcome are inherently challenging in terms of valid outcome measurement and ensuring adherence. Improvement in economic evaluation methodology relating specifically to ascertaining the economic burden of physical inactivity and the efficiency of effective interventions is needed before claims on resources can be established in an appropriate way. The additional component of adding an economic evaluation, while complicated, includes a critical component essential in clinical populations and healthcare decision-making and knowledge exchange.

Specifically, challenges in the broad area of sport and exercise medicine include adhering to the following four characteristics that a gold standard economic evaluation should include: (1) an intervention and a comparator commonly used in standard practice; (2) assess clinical outcomes as well as costs for intervention and comparator; (3) adequate power; (4a) sufficient follow-up time to assess full health gains or losses and (4b) an appropriate study time frame (ie, duration) to cover all main costs and health outcomes to aid in decision-making and adoption. ${ }^{30}$ These four characteristics often have different requirements for a didactic RCT compared with the concurrent economic evaluation. For example, the endpoint for the RCT primary outcome measure (eg, objective measure of physical activity) is often a shorter duration than the endpoint for the economic evaluation (eg, change in self-perceived health status). The above challenges are not specific only to trials with physical inactivity as a primary outcome-they are challenges that plague a number of populations and behavioural interventions. In addition, it is recommended to add Quality-Adjusted Life Years (QALYs) as an outcome to allow comparison between studies aimed at different outcomes of physical activity or behavioural change (see section Physical activity measurement for more details). Below we highlight methodological challenges that are specific to economic evaluation of trials with physical inactivity as a primary outcome. Finally, it is recommended to check the CHEERS guideline for reporting economic evaluations of health interventions that was recently published by ISPOR. ${ }^{31}$

A recent systematic review ascertained the cost of six different population-based interventions to examine the overall efficiency of these interventions at a population level using a lifetime time horizon. $^{28}$ The authors demonstrated that intervention programmes using pedometers and mass media-based community campaigns were the most cost-effective and potentially cost-saving strategies. Internet-based interventions, general practitioner physical activity prescription programmes and a programme encouraging more active transport also demonstrated potential to be cost effective. A physician referral to an exercise physiologist was the intervention that provided the least value for money. Importantly, conclusions from this systematic review need to be interpreted with caution because the economic analysis included intervention costs only. ${ }^{28} 32$ Healthcare costs, productivity or participants' opportunity costs often comprise the largest and most important part of the total costs. Where these costs differ between interventions, not taking them into account will give an incorrect ranking of the interventions. This methodological challenge needs rectifying as research on physical inactivity moves forward.

\section{Challenges in cost measurement}

Perspective and time horizon of a study determine which cost data should be identified. Preferably, economic evaluations should be carried out from a societal perspective which means that all costs should be taken into account no matter who pays or receives the benefit. All cost outcomes should be assessed for the timeframe of the study. ${ }^{26}$ Cost data might give insight in total costs, but also in shifts of costs over different groups of health expenditures. To reduce the burden for participants and researchers, cost data are often collected intermittently and subsequently extrapolated to the overall timeframe of the study. However, a recent study revealed that this intermittent data collection often results in bias for different categories of costs. Therefore, continuous data collection remains recommended. ${ }^{33}$

\section{Physical activity measurement}

One limitation affecting the accuracy of physical inactivity estimates is related to adequately defining 'physical inactivity' and its associated diseases. Self-reports of physical activity may be valid, but account for only a small percent of the variance of true physical activity. We now have objective devices for physical activity assessment, and these must be used more extensively in physical activity research. ${ }^{34}$ In addition, physical activity and fitness must be used more widely when evaluating the health effects and costs of other risk factors for non-communicable diseases. $^{35} 36$ For example, studies on obesity and health rarely account for objectively measured physical activity or physical fitness as a potential confounder. Thus, the burden of physical inactivity may be underestimated in these instances. 
As in other areas of physical inactivity research, health economics studies will benefit from the move to objective measures of physical inactivity and sedentary behaviour. Instruments that provide objective measurement include pedometers, accelerometers and devices such as ActivePAl or GPS which capture sitting behaviour. Beyond objective measurement, for CEA to be useful, we need to establish clinically meaningful changes in these objective measures for specific populations of interest. Without an accurate estimation of meaningful clinically important differences, the interpretability of CEA is severely limited. For example, cost per metabolic equivalent (MET) minute or MET hour would be a helpful item for CEAs (guideline=500 MET minutes). We know that cost-utility analysis is one method to move beyond this limitation; however, it has unique challenges that are discussed below.

Alternatively, a counter argument regarding the importance of accurately measuring physical inactivity can be debated if we instead measure the end goal of improving overall health. ${ }^{37}$ This argument places less importance on the accurate measurement of physical inactivity because ultimately, a successful physical inactivity intervention will lead to improved health outcomes that are measureable. Physical activity is an effective strategy for healthy aging as it reduces the incidence of cancer, diabetes and heart disease ${ }^{38}$; for example, an intervention aimed at physical activity promotion leading to better health outcomes that are measureable. If overall health outcomes improve, whether or not the intervention significantly decreased physical inactivity becomes less important. ${ }^{37}$ This perspective is most relevant when considering the health and economic benefits at a population level where the intervention is not tailored for specific subgroups but generalised to have positive impact at a population level. ${ }^{24} 39$

\section{Cost utility analyses using QALYs}

QALYs are a measure that is widely used in economic analyses of medical care interventions with several advantages, but there are specific challenges when using them to study prevention. QALYs can be derived through direct and indirect elicitation of patient preferences for specific health states over time. The advantage of QALYs is that they provide a common metric and basis for comparison of effectiveness of interventions across a diverse array of health conditions. As such, they can help place the effectiveness and efficiency of physical inactivity interventions in perspective with other diseases (ie, cancer and arthritis). One limitation of QALYs as an outcome measure in the primary prevention setting is that they are prone to having a 'ceiling effect' as the sample already has a relatively high QALY score at baseline. ${ }^{40}$ Furthermore, not all QALYs are equal, meaning that QALY's derived from different instruments and calculated by means of different algorithms are not directly comparable. ${ }^{41}$ Therefore, the method used to obtain health state utility values over time must be described and validated for the intended population, preferably also taking response shift into account.

\section{Section 6: Quality versus quantity}

To date, there exists health economic evidence for the burden of physical inactivity and the efficiency of various physical activity and exercise interventions. ${ }^{42} 43$ What remains missing is a comprehensive economic evaluation based on a high-quality clinical trial that measures key variables, is appropriately powered and included sufficient follow-up time for a meaningful economic endpoint. Hence, ideally, future studies will focus on the quality of the study design and economic evaluation methodology to provide an accurate estimate of burden of physical inactivity and efficiency of proposed physical activity and exercise interventions.
One of the reasons for the scant literature on CEAs of physical activity interventions seems to be that few research centres have the necessary transdisciplinary combination of public health, exercise science, economic, policy and behaviour change researchers with an infrastructure supporting collaboration. Explicitly creating several new multidisciplinary centres of excellence which can focus on CEAs may be useful for increasing research productivity in this area.

\section{Section 7: Interpretation and related action of present CEA evidence}

One noteworthy issue relating to CEA evidence is that often CEAs demonstrating better value for money (ie, less costly and more effective) are not implemented in society. The question remains-Why do CEAs that are more effective and less costly fail to get implemented? Below we detail a few explanations for the observed lack of action.

A key reason affecting implementation of cost-saving interventions in society relates to a methodological issue to which most CEAs succumb. There is a failure in current CEA evidence to consider the cost of investment and disinvestment in programmes. There is an opportunity cost to every decision. The opportunity cost of a choice is the value of the next best mutually exclusive alternative you choose to forgo. Hence, in order for CEA results to be usable for decision-making, they should include investment and/or disinvestment costs within the evaluation.

Another factor affecting implementation is that there are silos that often separate those who would need to invest in an intervention and those who ultimately would recoup the gains from the investment. To illustrate, if a local municipality (eg, City of Vancouver) invests in cycle paths that promote physical activity which leads to reduced healthcare costs, those savings would be received by the federal government which is responsible for the healthcare budget. Another example of two important silos that 'refuse' to discount between each other relates to investments for interventions is the healthcare silo versus a benefit in terms of reduced indirect cost losses in the workers compensation silo. Fragmented healthcare systems encourage 'silo thinking' whereas larger, integrated healthcare systems are more likely to wish to intervene at one point in the system (ie, in one silo) to reduce costs elsewhere (ie, in another silo). Of note, costing in different silos may have its own methodological challenges including identifying relevant and key cost items for each silo. An example of investment in preventive programmes that include physical activity is the Kaiser Permanente (California). Given that a compelling goal of our healthcare system is to achieve sustainable healthcare, a necessary first step is to bridge the gap between high-quality research, healthcare policy decision priorities and timelines and, investment and disinvestment opportunities.

Finally, an important factor potentially mitigating investment in health promotion is that the benefits are manifested a long time after the initial expenditures (ie, future discounting). Evidence-based examples include investing in child health ${ }^{44} 45$ with a view to reducing healthcare costs at a future point. One way of reporting the relative present cost and future saving could be by following the approach that Garnaut took when discussing climate change.

\section{CONCLUSIONS}

Physical inactivity is associated with a lower quality of life, including loss of function and development of non-communicable chronic diseases, compared to those leading physically active lives. People who are chronically physically inactive have a reduced 
Key messages

- Physical inactivity is a well-established problem.

- The economic burden of physical inactivity accounts for $1-3 \%$ of total healthcare costs annually. ${ }^{46}$

- There is high-quality evidence to suggest that physical inactivity can be mitigated; thus costs can be saved. ${ }^{17} 2627$

- It is currently difficult to determine how much can be saved. This is difficult to determine right now because of methodological challenges specific to conducting economic evaluations of physical inactivity interventions both at an individual and population level.

- The research priorities should be the following:

- Rectify the methodological challenges (ie, choice of comparator, adequate power, long enough time horizon to accurately depict health gains/losses, research questions that align with decision-making priorities, accurate assessment of physical inactivity outcomes and cost-utility analysis methodology) discussed in this article to generate high-quality economic evaluation data that will inform future health policy decisions.

- The economic group should reconvene, possibly in association with the IOC World Congress in Illness and Injury in Sport (2017) or the International Congress on Physical Activity and Public Health (ICPAPH) in 2016 contingent on funding support with the intent of building a consensus on guidelines for economic evaluations of effective physical inactivity interventions.

What are the new findings

- There is high quality evidence that physical inactivity can be mitigated thus saving health care costs.

- Methodological challenges for economic evaluations of physical inactivity interventions to date prevent accurate estimation of potential cost savings.

- Research priorities should be focused on rectifying methodological challenges and building a consensus on guidelines for economic evaluations of effective physical inactivity interventions. interventions to improve their activity levels. Most methodological challenges discussed relate specifically to accurately and objectively valuing wide ranging positive outcomes, determining reliable and clinically important differences in objective measures of physical inactivity, estimating investment and disinvestment costs and addressing barriers to implementation. Moving forward, we propose that guidelines specific for economic evaluations of physical inactivity and exercise intervention studies are developed to ensure accuracy and comparability of future economic evidence.

\section{Author affiliations}

${ }^{1}$ School of Population and Public Health, University of British Columbia, Vancouver, British Columbia, Canada

${ }^{2}$ Centre for Clinical Epidemiology and Evaluation, University of British Columbia, Vancouver, British Columbia, Canada

${ }^{3}$ Department of Public and Occupational Health \& EMGO+ Institute for Health and Care Research, VU University Medical Center, Amsterdam, The Netherlands

${ }^{4}$ Centre for Hip Health, Department of Physical Therapy, University of British Columbia, Vancouver, Canada

${ }^{5}$ Business and Economics, University of Melbourne, Australia

${ }^{6}$ Department of Kinesiology and Community Health, MC-588 University of Illinois at Urbana-Champaign, Illinois, USA

${ }^{7}$ Department of Human Movement Science, NUTRIM, School for Nutrition, Toxicology and Metabolism, Maastricht University, PO Box 616, 6200 MD

Maastricht, the Netherlands

${ }^{8}$ Institute of Sport, Exercise and Health, University College London Hospitals NHS

Foundation Trust, London, UK

${ }^{9}$ University of North Texas, Denton, Texas, USA

${ }^{10}$ Department of Exercise Science, University of South Carolina, Columbia, South

Carolina, South Carolina, USA

${ }^{11}$ NCCDPHP, Centers for Disease Control and Prevention, Atlanta, Georgia, USA

${ }^{12}$ Department of Experimental Medicine, University of British Columbia, Abbotsford, British Columbia, Canada

${ }^{13}$ Department of Family Practice, The University of British Columbia, Vancouver, British Columbia, Canada

${ }^{14}$ Aspetar, Orthopaedic and Sports Medicine Hospital, Doha, Qatar

Contributors JCD was responsible for study concept, writing of manuscript and critical review of the manuscript. EV, SB, TL-A, JB, DB, MH, RW, JRM, WVM, SNB, MP, JW, H-aT, EM and KMK were responsible for critical review of the manuscript.

Funding The Economics of Physical Inactivity Consensus (EPIC) Symposium was funded by the Canadian Institutes of Health Research-Mobility in Aging/CHHM Team in Vulnerable Seniors, BMJ Group, CHHM Walk the Talk Team. Canadian Institutes of Health Research (CIHR)-Emerging Team Grant in Mobility in Aging MAT-92025

Competing interests KMK was the Principle Investigator of the EPIC symposium. $J C D$ is funded by the Michael Smith Foundation for Health Research Postdoctoral Fellowship Award and the Canadian Institute for Health Research Postdoctoral Fellowship Award.

Provenance and peer review Not commissioned; externally peer reviewed.

\section{REFERENCES}

1 O'Reilly D, Gaebel K, Xie F, et al. Health economic evaluations help inform payers of the best use of scarce health care resources. Int J Circumpolar Health 2011;70:417-27

2 World Health Organization. http://www.euro.who.int/en/about-us/partners/observatory/ policy-briefs-and-summaries/promoting-health,-preventing-disease-is-there-an-economiccase (accessed 17 Feb 2014).

3 Global Advocacy for Physical Activity (GAPA) the Advocacy Council of the International Society for Physical Activity and Health (ISPAH). NCD prevention: investments that work for physical activity. 2011. http://www.globalpa.org.uk/ investmentsthatwork

4 Pettee Gabriel KK, Morrow JR Jr, Woolsey AL. Framework for physical activity as a complex and multidimensional behavior. J Phys Act Health 2012;9(Suppl 1): S11-18.

5 Lee I-M, Shiroma EJ, Lobelo F, et al. Effect of physical inactivity on major non-communicable diseases worldwide: an analysis of burden of disease and life expectancy. Lancet 2012;380:219-29.

6 Hallal PC, Andersen LB, Bull FC, et al. Global physical activity levels: surveillance progress, pitfalls, and prospects. Lancet 2012;380:247-57. ately understood, we first need to identify areas for methodological improvement when understanding the cost impact of inactivity and 
7 Teo K, Lear S, Islam S, et al. Prevalence of a healthy lifestyle among individuals with cardiovascular disease in high-, middle- and low-income countries: the Prospective Urban Rural Epidemiology (PURE) study. JAMA 2013;309:1613-21.

8 Bull FC, Bauman AE. Physical inactivity: the 'Cinderella' risk factor for noncommunicable disease prevention. J Health Commun 2011;16(Suppl 2):13-26.

9 Wing RR. Physical activity in the treatment of the adulthood overweight and obesity: current evidence and research issues. Med Sci Sports Exerc 1999;31: S547-52.

10 Al Tunaiji H, Davis J, Mackey D, et al. Population attributable fraction of type 2 diabetes due to physical inactivity in adults: a systematic review BMC public health. 2014; Revision 1 requested, 2014.

11 Janssen I. Health care costs of physical inactivity in Canadian adults. Appl Physiol Nutr Metab 2012;37:803-06.

12 Chief Medical Officer. On the state of the public health. Annual report of the chief medical officer 2009. http://webarchive.nationalarchives.gov.uk/20130107105354/ http://www.dh.gov.uk/prod_consum_dh/groups/dh_digitalassets/@dh/@en/@ps/ documents/digitalasset/dh_114012.pdf

13 Nyman JA. Productivity costs revisited: toward a New US Policy Health Economics 2012:21:1387-401.

14 Brown HE, Burton N, Gilson ND, et al. Measuring presenteeism: which questionnaire to use in physical activity research? J Phys Act Health 2014;11:241-8.

15 Noben CY, Evers SM, Nijhuis FJ, et al. Quality appraisal of generic self-reported instruments measuring health-related productivity changes: a systematic review. BMC Public Health 2014:14:115.

16 Brookmeyer R, Johnson E, Ziegler-Graham K, et al. Forecasting the global burden of Alzheimer's disease. Alzheimers Dement 2007:3:186-91.

17 Pratt M, Norris J, Lobelo F, et al. The cost of physical inactivity: moving into the 21st century. Br J Sports Med 2014:48:171-3.

18 Weiler $\mathrm{R}$, Feldschreiber $\mathrm{P}$, Stamatakis E. Medicolegal neglect? The case for physical activity promotion and exercise medicine. Br J Sports Med 2012;46:228-32.

19 Hillsdon M, Foster C, Thorogood M. Interventions for promoting physical activity. Cochrane Database Syst Rev 2005;(1):CD003180

20 Minton J, Dimairo M, Everson-Hock E, et al. Exploring the relationship between baseline physical activity levels and mortality reduction associated with increases in physical activity: a modelling study. BMJ Open 2013:3:e003509.

21 Wu S, Cohen D, Shi Y, et al. Economic analysis of physical activity interventions. Am J Prev Med 2011:40:149-58.

22 Müller-Riemenschneider F, Reinhold T, Willich SN. Cost-effectiveness of interventions promoting physical activity. Br J Sports Med 2009;43:70-6.

23 Cecchini M, Sassi F, Lauer JA, et al. Tackling of unhealthy diets, physical inactivity, and obesity: health effects and cost-effectiveness. Lancet 2010;376:1775-84.

24 Garrett S, Elley CR, Rose SB, et al. Are physical activity interventions in primary care and the community cost-effective? A systematic review of the evidence. $\mathrm{Br} J \mathrm{Gen}$ Pract 2011:61:e125-33.

25 Stamatakis $E$, Weiler R. Prevention of cardiovascular disease: why do we neglect the most potent intervention? Heart 2010:96:261-2.

26 Roux L, Pratt M, Tengs TO, et al. Cost effectiveness of community-based physical activity interventions. Am J Prev Med 2008;35:578-88.

27 Muller-Riemenschneider F, Reinhold T, Willich SN. Cost-effectiveness of interventions promoting physical activity. Br J Sports Med 2009;43:70-6.
28 Cobiac LJ, Vos T, Barendregt JJ. Cost-effectiveness of interventions to promote physical activity: a modelling study. PLoS Med 2009;6:e1000110.

29 Davis JC, Robertson MC, Ashe MC, et al. Does a home-based strength and balance programme in people aged $>$ or $=80$ years provide the best value for money to prevent falls? A systematic review of economic evaluations of falls prevention interventions. Br J Sports Med 2010;44:80-9.

30 Glick HA, Doshi JA, Sonnad SA, et al. Economic evaluation in clinical trials. New York, USA: Oxford University Press, 2007.

31 Husereau D, Drummond M, Petrou S, et al. Consolidated Health Economic Evaluation Reporting Standards (CHEERS) statement. Int I Technol Assess Health Care 2013;29:117-22

32 Pavey TG, Anokye N, Taylor AH, et al. The clinical effectiveness and cost-effectiveness of exercise referral schemes: a systematic review and economic evaluation. Health Technol Assess 2011;15:i-xii, 1-254.

33 Hendriks MR, Al MJ, Bleijlevens MH, et al. Continuous versus intermittent data collection of health care utilization. Med Decis Making 2013;33: 998-1008

34 Banda JA, Hutto B, Feeney A, et al. Comparing physical activity measures in a diverse group of midlife and older adults. Med Sci Sports Exerc 2010;42:2251-7.

35 Swift DL, Lavie CJ, Johannsen NM, et al. Physical activity, cardiorespiratory fitness, and exercise training in primary and secondary coronary prevention. Circ $J$ 2013;77:281-92

36 Barry VW, Baruth $\mathrm{M}$, Beets MW, et al. Fitness vs. fatness on all-cause mortality: a meta-analysis. Prog Cardiovasc Dis 2014;56:382-90.

37 Cobiac LJ, Vos T, Barendregt JJ. Cost-effectiveness of interventions to promote physical activity: a modelling study. PLoS Med 2009;6:e1000110.

38 Booth FW, Gordon SE, Carlson CJ, et al. Waging war on modern chronic diseases: primary prevention through exercise biology. J App/ Physiol 2000;88:774-87.

39 Wu S, Cohen D, Shi Y, et al. Economic analysis of physical activity interventions. Am J Prev Med 2011:40:149-58.

40 Tordrup D, Mossman J, Kanavos P. Responsiveness of the Eq-5d to clinical change: is the patient experience adequately represented? Int J Technol Assess Health Care 2014:1-10. [Epub ahead of print]

41 Marra CA, Marion SA, Guh DP, et al. Not all 'quality-adjusted life years' are equal. J Clin Epidemiol 2007;60:616-24.

42 van Dongen JM, Proper $\mathrm{Kl}$, van Wier MF, et al. Systematic review on the financial return of worksite health promotion programmes aimed at improving nutrition and/ or increasing physical activity. Obes Rev 2011;12:1031-49.

43 van Dongen JM, Proper Kl, van Wier MF, et al. A systematic review of the cost-effectiveness of worksite physical activity and/or nutrition programs. Scand J Work Environ Health 2012:38:393-408.

44 Drummond MF, Sculpher MJ, Torrance GW, et al. Methods for the economic evaluation for health care programmes. 3rd edn. New York, USA: Oxford University Press, 2005

45 Siegrist $\mathrm{M}$, Hanssen $\mathrm{H}$, Lammel $\mathrm{C}$, et al. A cluster randomised school-based lifestyle intervention programme for the prevention of childhood obesity and related early cardiovascular disease (JuvenTUM 3). BMC Public Health 2011;11:258.

46 Draper $C E$, de Villiers A, Lambert EV, et al. HealthKick: a nutrition and physical activity intervention for primary schools in low-income settings. BMC Public Health 2010;10:398 